\title{
Influence of organic solvent treatment on elasticoluminescent property of europium-doped strontium aluminates
}

\author{
Yuki Fujio ${ }^{\mathrm{a},{ }^{*}}$, Chao-Nan $\mathrm{Xu}^{\mathrm{a}, \mathrm{b},{ }^{*}, \text { Nao Terasaki }}{ }^{\mathrm{a}}$, and Naohiro Ueno ${ }^{\mathrm{a}, \uparrow}$ \\ ${ }^{a}$ Measurement Solution Research Center, National Institute of Advanced Industrial Science \\ and Technology (AIST), Saga 841-0052, Japan \\ ${ }^{b}$ International Institute for Carbon-Neutral Energy Research (WPI-I $\left.{ }^{2} C N E R\right)$, Kyushu \\ University, Fukuoka 819-0395, Japan
}

Keywords: mechanoluminescence; $\mathrm{SrAl}_{2} \mathrm{O}_{4}: \mathrm{Eu}$; strain measurement

$\dagger$ Present address: Graduate School of Science and Engineering, Saga University, Saga

840-8502, Japan

${ }^{*}$ Corresponding author: Tel.: +81-942-81-4011; Fax: +81-942-81-3696

E-mail address: yuki-fujio(at)aist.go.jp (Y. Fujio), cn-xu(at)aist.go.jp (C. N. Xu) 


\begin{abstract}
The influence of an organic solvent treatment on elasticoluminescent (ELS) characteristics of mechanoluminescent (ML) sensor using the composite film consisting of an ELS material and epoxy resin was investigated. We used strontium aluminate doped with a small amount of europium $\left(\mathrm{SrAl}_{2} \mathrm{O}_{4}: \mathrm{Eu}, \mathrm{SAOE}\right)$ as an ELS material in this study. After evaluating the ELS characteristics of the fabricated ML sensors using SAOE treated with/without various organic solvents, SAOE treated with methanol and ethanol showed lower ELS intensities than that of untreated SAOE. In contrast, the ELS response curves against strain for the ML sensors using SAOE treated with acetone and toluene, overlapped with that of untreated SAOE. From the characterization of SAOE treated with alcohols, such as methanol and ethanol, we can hypothesize that poor ELS characteristics is due to the degradation of the SAOE grain surfaces by the hydrolyze reaction of SAOE with hydroxyl group of alcohol. Thus, on the basis of the obtained results, we can conclude that the selection of organic solvent used in the preparation of SAOE film is of considerable importance in the development of ML sensor with a highly-reliable ELS characteristic.
\end{abstract}




\section{Introduction}

Recently the structural health monitoring of various constructions, such as bridge, building, chemical plant, and social infrastructure, is a very important issue. Actually, there have been a lot of accidents caused by age-related degradation all over the world, for instance, the collapses of the Mississippi river bridge in Minneapolis, USA (August 1, 2007) and Sasago tunnel in Yamanashi, Japan (December 2, 2012). In order to ensure the safety in the social life, a development of a highly-reliable structural health monitoring technique is an urgent and crucible concern.

So far, there have been numerous reports in regard to the sensing technology, such as an ultrasonic wave method [1], electrical strain gage [2], fiber optic sensor [3,4], and so on, aiming at a structural health monitoring application. Quite recently, we have reported that the mechanoluminescent (ML) sensor using a composite film consisting of an ML material and organic compounds can be useful technique for not only a dynamic visualization of the strain (or stress) distribution in complicated structure of metal substrate [5] but also a health monitoring of an aged bridge [6]. The ML is the phenomenon of luminescence induced by mechanical actions, such as compression, tension, friction, or torsion. In the ML sensor, an elasticoluminescence (ELS) is used as a sensing signal for the reason of a reversible luminescence in the elastic deformation and its proportionality to the strain (or stress). The detail of ELS definition was mentioned in a paper [7]. Among various reports about the ELS materials aiming at strong luminescence and various emission colors (wavelength), for example, $\mathrm{ZnS}: \mathrm{Mn} \quad[8,9], \quad \mathrm{CaAl}_{2} \mathrm{Si}_{2} \mathrm{O}_{8}: \mathrm{Eu} \quad[10], \quad \mathrm{BaTiO}_{3}-\mathrm{CaTiO}_{3}: \operatorname{Pr} \quad[11,12]$ and $\mathrm{Sr}_{n+1} \mathrm{Sn}_{n} \mathrm{O}_{3 n+1}: \mathrm{Sm}$ [13], strontium aluminates doped with a small amount of europium $\left(\mathrm{SrAl}_{2} \mathrm{O}_{4}: \mathrm{Eu}, \mathrm{SAOE}\right)$ is one of the promising ELS material owing to the strongest visible light emission [14-21]. From the application point of ELS material to the ML sensor which consists of ELS material and organic compounds [22-25], it is vital to choose the appropriate organic 
compounds, which are polymeric materials and organic solvents, to the ELS material, keeping or enhancing the original ELS characteristics. However, the effect of organic compounds on the ELS characteristics has been still unclear, although a selection of the combination of an ELS material and organic materials is the most important for achieving a high performance ELS characteristics.

In this study, we fabricated ML sensors using composite films of SAOE powder treated with/without organic solvents and epoxy resin, and investigated their ELS characteristics. As an organic solvent, we examined four kinds of solvents with different polarities, such as methanol, ethanol, acetone, and toluene. After evaluating the ELS characteristics of the prepared SAOE films, other optical and structural characteristics were also examined.

\section{Experimental}

\subsection{Preparation of SAOE and SAOE treated with organic solvent}

The SAOE powder was synthesized through a conventional solid-state reaction method. The commercial $\mathrm{SrCO}_{3}$ (Kanto Chemical Co., Inc., Japan), $\alpha-\mathrm{Al}_{2} \mathrm{O}_{3}$, and $\mathrm{Eu}_{2} \mathrm{O}_{3}$ (Kojundo Chemical Laboratory Co., Ltd, Japan) were weighed precisely to the molar ratios of $\mathrm{Sr}_{0.97} \mathrm{Al}_{2} \mathrm{O}_{4}: \mathrm{Eu}_{0.03}$, and mixed together by means of ball-milling method using zirconia balls ( $5 \mathrm{~mm}$ in diameter) in ethanol solution at $100 \mathrm{rpm}$ for $20 \mathrm{~h}$. The resulting suspension was dried to vaporize an ethanol on an electrical hot plate, stirring continually, and pre-calcined at $800^{\circ} \mathrm{C}$ for $1 \mathrm{~h}$ in air. The solidified product was ground in a mortar and sintered again at $1350^{\circ} \mathrm{C}$ for $4 \mathrm{~h}$ in $5 \% \mathrm{H}_{2} / \mathrm{Ar}$ atmosphere. The final SAOE powder was obtained by thoroughly pulverizing the sintered powder in a mortar.

The synthesized SAOE powder (3.0 g) was vigorously stirred for $24 \mathrm{~h}$ in each of four kinds of organic solvent (100 ml each). The obtained suspensions were dried to obtain SAOE 
treated with an organic solvent. The each of SAOE powders treated with methanol, ethanol, acetone, and toluene, denoted by SAOE-Mt, SAOE-Et, SAOE-Ac, and SAOE-To, respectively.

\subsection{Evaluation of ELS characteristics}

The SAOE films were fabricated by means of screen printing technique using a mixture of an SAOE powder and epoxy resin. In all cases, the thicknesses of the prepared SAOE films were approximately $60 \mu \mathrm{m}$. The fabricated SAOE films adhered to the front side of SUS631 substrate with a commercial adhesive. The commercial strain gage was also applied on the back side of the SUS631 substrate. The ELS characteristics of the prepared SAOE films were evaluated under an ELS evaluation system in a dark room at room temperature. This system was built up with four parts; (1) a metal substrate (SUS631, $\left.250 * 20 * 0.5^{\mathrm{t}} \mathrm{mm}\right)$ attached with the SAOE film $(10 * 10 \mathrm{~mm})$ and a commercial strain gage (Kyowa Electronic Instruments Co., Ltd., Japan), to record ELS images and strain simultaneously, (2) material testing machine (MTS 810, MTS Systems Co., US), to apply a mechanical load to the metal substrate attached with the SAOE film and strain gage, (3) a CCD camera to take ELS images, and (4) a computer, to control a material testing machine and record ELS images and strains, as has already been reported elsewhere $[5,26]$. For reproducible and quantitative ELS measurement, the ML sensor applied on the SUS substrate was once irradiated by ultraviolet light $\left(\mathrm{UV}, 365 \mathrm{~nm}, 0.7 \mathrm{~mW} / \mathrm{cm}^{2}\right)$ for $1 \mathrm{~min}$ and keep under dark condition for $1 \mathrm{~min}$. The ELS intensities corresponding to strain were estimated from the obtained ELS images and strain gage.

\subsection{Characterization SAOE materials}

The crystal structure and microstructure of the prepared SAOE powders were elucidated via X-ray diffraction (XRD, RINT2000, Rigaku Co., Japan) analysis using Cu K $\alpha$ radiation $(\lambda=1.5418 \AA ; 40 \mathrm{kV}, 20 \mathrm{~mA})$ and field-emission scanning electron microscopy 
(FE-SEM, JSM-7001F, JEOL Ltd., Japan), respectively. The photoluminescence (PL) emission and excitation spectra were measured by using a spectrofluorometer (FP-6600, JASCO Co., Japan). The PL emission spectra were recorded in the range of 400-700 nm under exposure of $365 \mathrm{~nm}$, and the PL excitation spectra were monitored at $514 \mathrm{~nm}$ in the excitation wavelength range of $270-500 \mathrm{~nm}$. The measurements for a thermoluminescence $(\mathrm{ThL})$ glow curves were performed by means of a system built up with the above mentioned spectrofluorometer (FP-6600) and a heating/freezing stage (Linkam Scientific Instruments Ltd., UK). Weighed amount of each of prepared SAOE powders (200 $\mathrm{mg}$ each) was set in a metal holder. It was heated up to $300^{\circ} \mathrm{C}$ on the heating/freezing stage to release all carriers trapped in defect inside $\mathrm{SrAl}_{2} \mathrm{O}_{4}$ matrix, and then cooled down to room temperature $\left(27^{\circ} \mathrm{C}\right)$. After exposure of UV light to SAOE powder for $5 \mathrm{~min}$ and waiting for $1 \mathrm{~min}$, it was heated up to $300^{\circ} \mathrm{C}$ at the heating rate of $30^{\circ} \mathrm{C} / \mathrm{min}$ to record the $\mathrm{ThL}$ glow curves.

\section{Results and discussion}

\subsection{Influence of organic solvent treatments on ELS characteristics}

The influence of different organic solvent treatment of SAOE powder on ELS characteristics of ML sensors was investigated by measuring ELS intensities corresponding to strain under the ELS evaluation system. Before comparison of ELS characteristics of the prepared SAOE films, the ELS characteristics of SAOE film, which was prepared by using SAOE powder without treatment of any organic solvent, were evaluated and shown in Fig. 1(a). Figure 1(a) shows the ELS response curve of the ML sensor using SAOE film as well as strain curve of the attached strain gage, when a tensile load was applied to the specimen. In this study, the applied tensile load and loading speed were controlled to be approximately $1500 \mu$ st of strain and $1100 \mu \mathrm{st} / \mathrm{sec}$ of strain rate, respectively. It is seen that the ELS intensity of ML sensor using SAOE film increases with increasing strain of metal 
substrate, confirming that the fabricated ML sensor is used as the strain measurement technology. Figure 1(b) depicts the comparison of strain dependency of ELS intensities of ML sensors using SAOE-Mt, SAOE-Et, SAOE-Ac, and SAOE-To films. For comparison, a result for the ML sensor using a SAOE film was also shown in Fig. 1(b). It was found that the ELS intensities of SAOE-Mt and SAOE-Et films decreased in comparison with that of SAOE film, especially the ELS intensity declined significantly by a methanol treatment. In contrast, the ELS characteristics of SAOE-Ac and SAOE-To overlapped with that of SAOE film over the examined strain range. Thus, this result indicates that there is almost no influence of acetone and toluene treatments on an ELS characteristic of the ML sensor using SAOE film, suggesting that acetone and toluene can be used as an organic solvent in fabrication of an ML sensor. Unfortunately, the treatments of methanol and ethanol, which are type of alcohol, adversely affect the ELS characteristics of ML sensor using SAOE powders.

\subsection{Characterization of SAOE powders}

In order to clarify the cause of influence of alcohol treatments on the ELS characteristics, the crystal structure of these SAOE powders were investigated via XRD and the observed diffraction patterns are given in Fig. 2(a). The diffraction patterns of SAOE, SAOE-Ac, and SAOE-To powders were also shown in same figure. As can be seen from XRD patterns of SAOE powder, all the diffraction peaks coincide with those of the monoclinic $\mathrm{SrAl}_{2} \mathrm{O}_{4}$ phase (PDF No.: 01-074-0794 in ICDD), with no other impurity phases being detectable. The SAOE-Ac and SAOE-To powders were also observed only monoclinic $\mathrm{SrAl}_{2} \mathrm{O}_{4}$ phase. Contrastively, the XRD patterns of SAOE-Mt and SAOE-Et powders were discerned tiny peaks at around 25 degree, although the monoclinic $\mathrm{SrAl}_{2} \mathrm{O}_{4}$ phase remained in both diffraction patterns. Figure 2(b) depicts the magnified XRD patterns of SAOE, SAOE-Mt, and SAOE-Et. It is clearly seen that the tiny peaks appear at around 25 degree in XRD patterns of SAOE-Mt and SAOE-Et. These tiny peaks were unable to be attributed to 
any possible compounds.

The morphologies of prepared SAOE powders were also observed by means of FE-SEM and the obtained SEM images are presented in Fig. 3. The SEM image of untreated SAOE powder (Fig. 3(a)) showed that SAOE grains have smooth surface and they were agglutinated to form much larger secondary particles after sintering at $1350^{\circ} \mathrm{C}$ for $4 \mathrm{~h}$ in $\mathrm{H}_{2} / \mathrm{Ar}$ atmosphere. After treatments of methanol and ethanol, it was seen that a massive amount of solids clung to SAOE grains in Figs. 3(b) and (c). While, in the case of acetone and toluene treatments (Figs. 3(d) and (e)), the number of solids attached on SAOE grains were significantly lower. Here, it has been reported that $\mathrm{SrAl}_{2} \mathrm{O}_{4}$ was easy to metamorphose and change into other compounds in $\mathrm{H}_{2} \mathrm{O}$ [27-30]. In consequence of only the immersion in deionized water, a hydrolyze reaction of $\mathrm{SrAl}_{2} \mathrm{O}_{4}$ with hydroxide ion proceeds and an equilibrium reaction between $\mathrm{Sr}_{3} \mathrm{Al}_{2}(\mathrm{OH})_{12}$ and $\left[\mathrm{Sr}_{3} \mathrm{Al}_{2}(\mathrm{OH})_{11}\right]^{+}$occurred [27]. In addition, the different composition of strontium aluminates, such as $\mathrm{SrAl}_{4} \mathrm{O}_{7}, \mathrm{SrAl}_{12} \mathrm{O}_{19}$, and $\mathrm{Sr}_{4} \mathrm{Al}_{14} \mathrm{O}_{25}$, were also produced [29]. Based on these reports, we can speculate that the SAOE grains react with the hydroxyl group of alcohol during alcohol treatments and produce hydrolysates or the different composition of strontium aluminates onto the SAOE grains. Thus, the appeared new peaks at around 25 degree in diffraction patterns of SAOE-Mt and SAOE-Et powders seem to derive from a hydrolysate or different strontium aluminates. This hypothesis supports that the ELS characteristics of SAOE-Mt film is lower than that of SAOE-Et film, since the number of hydroxyl group in methanol is larger than that in ethanol when compared to same amount of solution $(100 \mathrm{ml})$.

The PL emission and excitation spectra for SAOE powders treated with/without various organic solvents were also measured and compared (not shown here). The peak positions of PL emission and excitation spectra for the SAOE powders treated with organic solvents were in accordance with those of untreated SAOE powder. This indicates that the 
chemical composition and structure of $\mathrm{SrAl}_{2} \mathrm{O}_{4}$ matrix as well as chemical state of $\mathrm{Eu}^{2+}$ ions are mostly unchanged, excluding morphology and chemical composition of some part of SAOE grains surface.

Considering the ELS mechanism which is related to carrier trapping model [31], an ELS characteristic is considered to be deeply linked with carrier captured in the traps derived from the lattice defects in $\mathrm{SrAl}_{2} \mathrm{O}_{4}$ matrix. In order to evaluate the state of carrier inside the prepared SAOE powders, the measurements of ThL glow curves were carried out. Figure 4 shows a comparison of ThL glow curves for the prepared SAOE powders in the temperature range of $27-300^{\circ} \mathrm{C}$ at the heating rate of $30^{\circ} \mathrm{C} / \mathrm{min}$. In all the measurement, UV light $(365 \mathrm{~nm})$ was exposed to samples for $5 \mathrm{~min}$ and wait for $1 \mathrm{~min}$ before starting the measurements. It can be seen that there are mainly two broadened peaks at around 50 and $100^{\circ} \mathrm{C}$ in all of prepared SAOE powders. It is noted that the position of a peak at around $100^{\circ} \mathrm{C}$ for SAOE-Mt and SAOE-Et powders slightly shifts to higher temperature side compared to untreated SAOE powder, although the magnitude of peak shift is not so large, pointing out that it is difficult to release the trapped carriers from the defect. Based on these results obtained in this study, therefore, we can conclude that the decrease in ELS characteristics of SAOE powder by alcohol treatment is due to the formation of deeper carrier trapped level, which was generated by some chemical reactions on the SAOE grains surface, though the details should be examined further.

\section{Conclusions}

The ELS characteristics of the ML sensor using an SAOE film consisting of SAOE powder and an epoxy resin were degraded by the alcohol treatment, such as a stirring SAOE powder in methanol or ethanol before mixing with epoxy resin to prepare SAOE film. This is

presumably due to an appearance of hydrolysates or different chemical composition of 
strontium aluminates onto SAOE grain produced by chemical reactions between $\mathrm{SrAl}_{2} \mathrm{O}_{4}$ and hydroxyl group in alcohol solution. This indicates that the selection of an appropriate organic solvent in the sensing film preparation of ML sensor is essential in view of preventing a decrease as well as a further increase in ELS characteristics of ML sensor.

\section{Acknowledgements}

The authors would like to thank Dr. Akiyama, Dr. Yamada, Dr. Uehara, Ms. Kawasaki, and the all member of Advanced Integrated Sensing team of AIST for their valuable discussions and help in the experiment. This work was partially supported by Adaptable and Seamless Technology Transfer Program through Exploratory Research, Japan Science and Technology Agency as well as Grant-in-Aid for Scientific Research (A) (Grant number: 25249100) from Japan Society for the Promotion of Science. 


\section{References}

[1] Y. Lu, J.E. Michaels, Ultrasonics 43 (2005) 717.

[2] I. Kang, M.J. Schulz, J.H. Kim, V. Shanov, D. Shi, Smart Mater. Struct. 15 (2006) 737.

[3] H.N. Li, D.S. Li, G.B. Song, Eng. Struct. 26 (2004) 1647.

[4] J.M. Henault, M. Quiertant, S. Delepine-Lesoille, J. Salin, G. Moreau, F. Taillade, K. Benzarti, Constr. Build. Mater. 37 (2012) 916.

[5] C. Li, C.N. Xu, L. Zhang, H. Yamada, Y. Imai, J. Vis. 11(4) (2008) 329.

[6] N. Terasaki, C.N. Xu, C. Li, L. Zhang, C. Li, D. Ono, M. Tsubai, Y. Adachi, Y. Imai, N. Ueno T. Shinokawa, Proceedings of SPIE 8348 (2012) 83482D.

[7] C.N. Xu, H. Yamada, X. Wang, X.G. Zheng, Appl. Phys. Lett. 84 (2004) 3040.

[8] C.N. Xu, T. Watanabe, M. Akiyama, X.G. Zheng, Appl. Phys. Lett. 74 (1999) 1236.

[9] B.P. Chandra, C.N. Xu, H. Yamada, X.G. Zheng, J. Lumines. 130 (2010) 442.

[10] L. Zhang, H. Yamada, Y. Imai, C.N. Xu, J. Electrochem. Soc. 155(3) (2008) J63.

[11] J.C. Zhang, X. Wang, X. Yao, C.N. Xu, H. Yamada, J. Electrochem. Soc. 157(12) (2010) G269.

[12] J.C. Zhang, M. Tang, X. Wang, Y. Li, X. Yao, Ceram. Int. 38S (2012) S581.

[13] S. Kamimura, H. Yamada, C.N. Xu, Appl. Phys. Lett. 101 (2012) 091113.

[14] C.N. Xu, T. Watanabe, M. Akiyama, X.G. Zheng, Appl. Phys. Lett. 74 (1999) 2414.

[15] C.N. Xu, H. Yamada, X. Wang, X.G. Zheng, Appl. Phys. Lett. 84 (2004) 3040.

[16] K.S. Sohn, D.H. Park, J.S. Kim, J. Electrochem. Soc. 152 (2005) H161.

[17] J.S. Kim, Y.N. Kwon, N. Shin, K.S. Sohn, Appl. Phys. Lett. 90 (2007) 241916.

[18] X. Fu, H. Yamada, C.N. Xu, J. Electrochem. Soc. 156(9) (2009) J249.

[19] S. Someya, K. Ishii, M. Saeki, T. Munakata, Opt. Lett. 38(7) (2013) 1095.

[20] V.K. Chandra, B.P. Chandra, P. Jha, Appl. Phys. Lett. 102 (2013) 241105.

[21] G.J. Yun, M.R. Rahimi, A.H. Gandomi, G.C. Lim, J.S. Choi, Smart Mater. Struct. 22 
(2013) 055006.

[22] J.S. Kim, Y.N. Kwon, K.S. Sohn, Acta Mater., 51(20) (2003) 6437.

[23] J.S. Kim, Y.N. Kwon, N.S. Shin, K.S. Sohn. Acta Mater., 53(16) (2005) 4337.

[24] Y. Imai, R. Momoda, C.N. Xu, Mater. Lett., 61 (2007) 4124.

[25] S. Timilsina, K.H. Lee, I.Y. Jang, J.S. Kim, Acta Mater., 61(19) (2013) 7197.

[26] C. Li, C.N. Xu, Y. Imai, N. Bu, Strain 47 (2011) 483.

[27] J. Lin, Y. Huang, J. Zhang, F. Shi, S. Wei, J. Gao, Z. Huang, X. Ding, C. Tang, Mater. Chem. Phys. 108 (2008) 440.

[28] Y. Imai, R. Momoda, Y. Adachi, K. Nishikubo, Y. Kaida, H. Yamada, C.N. Xu, J. Electrochem. Soc. 154(3) (2007) J77.

[29] C. Guo, L. Luan, D. Huang, Q. Su, Y. Lv, Mater. Chem. Phys. 106 (2007) 268.

[30] L. Zhang, H. Yamada, Y. Imai, C.N. Xu, J. Electrochem. Soc. 155(3) (2008) J63.

[31] C.N. Xu, Encyclopedia of Smart Materials, Edited by M. Schwartz. John Wiley \& Sons Inc., New York, 1 (2002) 190. 
Figure captions

Fig. 1 (a) ELS response curve of ML sensor using untreated SAOE film and strain curve of strain gage after UV light exposure for $1 \mathrm{~min}$ and wait for 1min; (b) comparison of strain dependency of ELS intensities for ML sensors using SAOE, SAOE-Mt, SAOE-Et, SAOE-Ac, and SAOE-To films.

Fig. 2 (a) XRD patterns of SAOE, SAOE-Mt, SAOE-Et, SAOE-Ac, and SAOE-To powders as well as standard PDF data for $\mathrm{SrAl}_{2} \mathrm{O}_{4}$ (01-074-0794), and (b) magnified XRD patterns of SAOE, SAOE-Mt, and SAOE-Et powders.

Fig. 3 Representative SEM images of (a) SAOE, (b) SAOE-Mt, (c) SAOE-Et, (d) SAOE-Ac, and (e) SAOE-To powders.

Fig. $4 \mathrm{ThL}$ glow curves in the examined range of $27-300^{\circ} \mathrm{C}$ at heating rate of $30^{\circ} \mathrm{C} / \mathrm{min}$ for (a) SAOE, (b) SAOE-Mt, (c) SAOE-Et, (d) SAOE-Ac, and (e) SAOE-To powders. 
Y. Fujio et al., Fig. 1
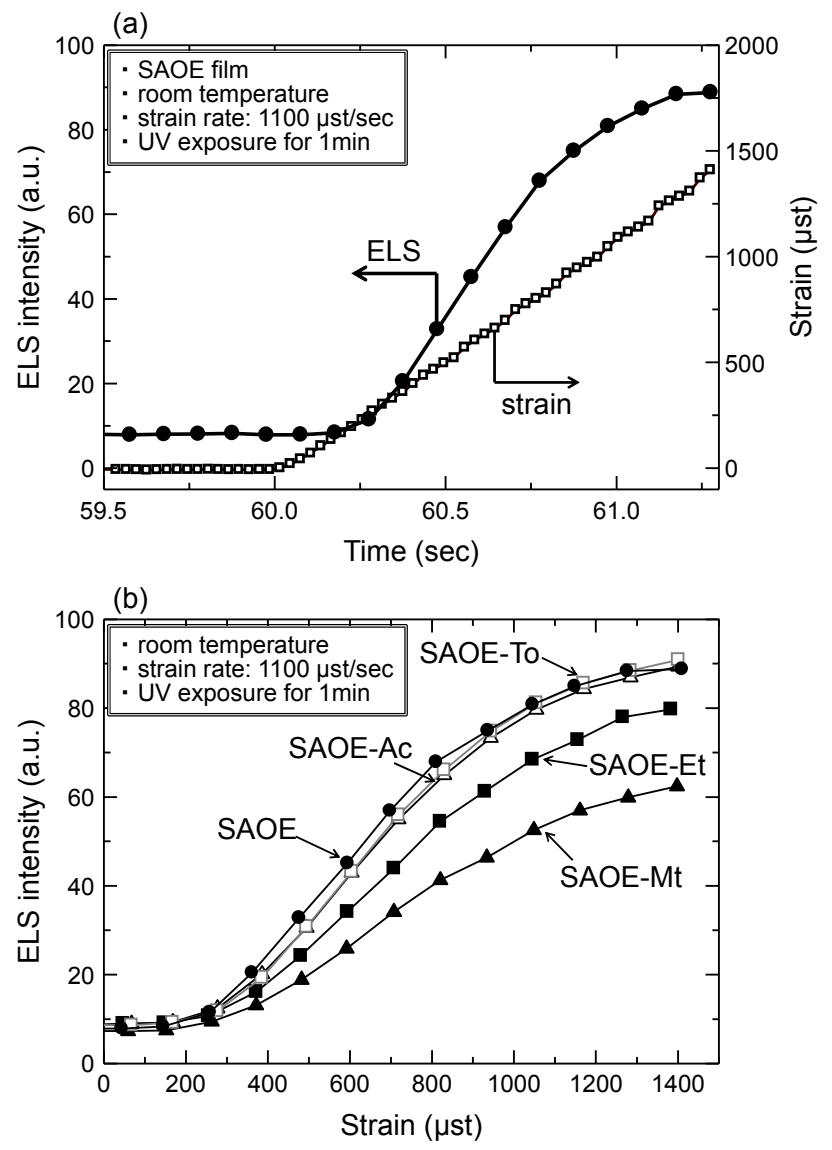
Y. Fujio et al., Fig. 2
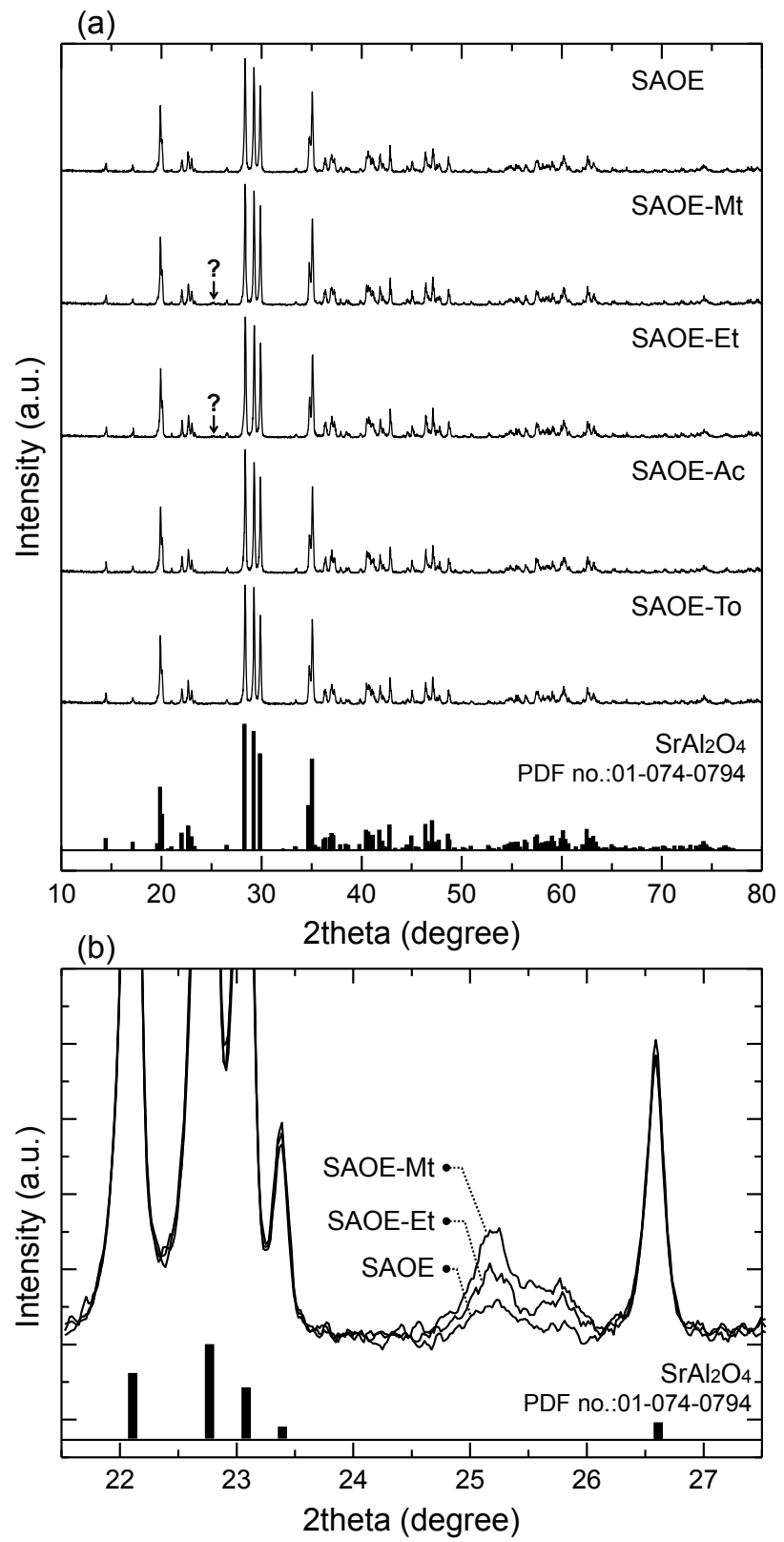
Y. Fujio et al., Fig. 3

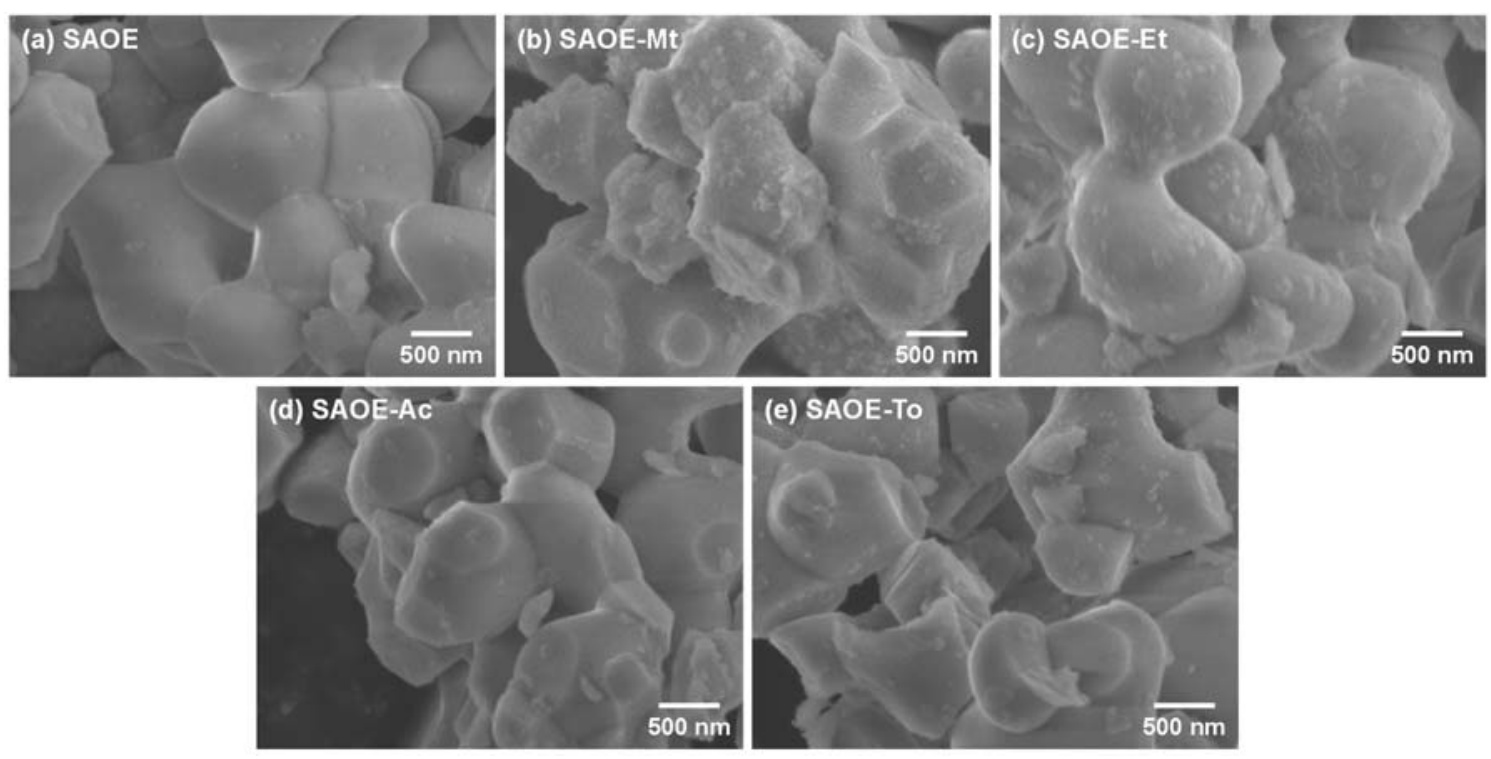


Y. Fujio et al., Fig. 4

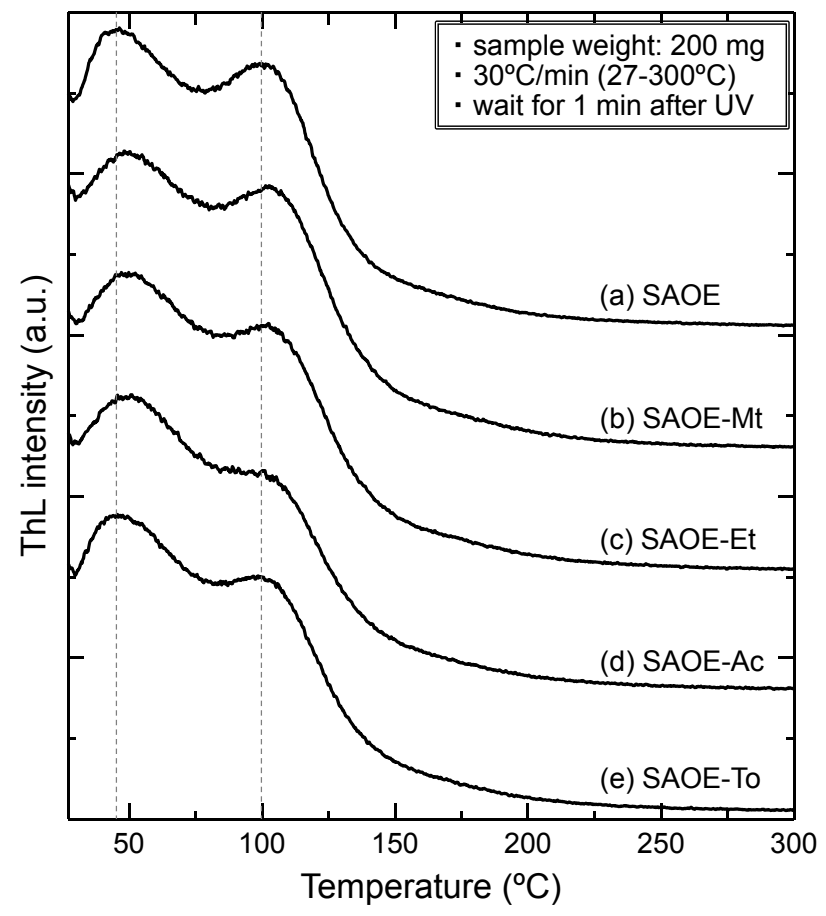

